\title{
Magnetohydrodynamic Impact on Carreau Thin Film Couple Stress Nanofluid Flow over an Unsteady Stretching Sheet
}

\author{
Taza Gul, ${ }^{1,2}$ Muhammad Rehman, ${ }^{1}$ Anwar Saeed, ${ }^{3}$ Imran Khan, ${ }^{4}$ Amir Khan, ${ }^{5}$ \\ Saleem Nasir, ${ }^{1}$ and Abdul Bariq ${ }^{6}{ }^{6}$ \\ ${ }^{1}$ City University of Science and Information Technology, Peshawar 25000, KP, Pakistan \\ ${ }^{2}$ Higher Education Archives \& Libraries Department KP, Government Superior Science College, Peshawar, Pakistan \\ ${ }^{3}$ Department of Mathematics, Abdul Wali Khan University, Mardan 23200, Pakistan \\ ${ }^{4}$ Department of Mathematics, Bacha Khan University, Charsadda 24450, Pakistan \\ ${ }^{5}$ Department of Mathematics and Statistics, University of Swat, Mingora 19200, KP, Pakistan \\ ${ }^{6}$ Department of Mathematics, Laghman University, Mehterlam 2701, Laghman, Afghanistan \\ Correspondence should be addressed to Abdul Bariq; abdulbariq.maths@lu.edu.af
}

Received 19 July 2021; Revised 24 August 2021; Accepted 22 September 2021; Published 30 September 2021

Academic Editor: Tirivanhu Chinyoka

Copyright ( $\odot 2021$ Taza Gul et al. This is an open access article distributed under the Creative Commons Attribution License, which permits unrestricted use, distribution, and reproduction in any medium, provided the original work is properly cited.

\begin{abstract}
A mathematical model of time-dependent thin-film flow of Carreau liquid over a stretching surface is studied in this investigation in the presence of couple stress and uniform magnetic field. To explain the properties of heat and mass transport phenomena, the influence of both thermophoresis and Brownian motion variables is used. For the conversion of the model framework (momentum, heat, and concentration expression with boundary conditions) into a set of ordinary differential equations, the appropriate transformation technique is followed. By using analytical tool, HomotopyAnalysis Method (HAM), the transformed model expressions are solved. For different estimations of the affected physical factors, the numerical results involving skinfriction coefficient, Nusselt number, Sherwood number, fluid velocity profile, thermal profile, and concentration profile are displayed graphically. Besides, the findings for skin-friction coefficient, $\mathrm{Nu}_{x}$, and $\phi(\eta)$ are given in the table format. In raising the fluid temperature, the effect of thermophoresis and magnetic parameters is beneficial. With the Brownian motion and Schmidt number, the solute concentration is found to reduce.
\end{abstract}

\section{Introduction}

The thin-film liquid has numerous applications in various sectors such as coating industries, oil recovery, colloid suspension, soap bubbles, and interface sciences. The analysis of the stretching phenomena with heat and mass transfer together with the thin liquid film has dynamic usages. In mechanics, there are some nonlinear issues open towards complete mathematical learning. Heat transfer remains an essential portion of individual parts such as chemical production, atomic energy, and lubrication. NonNewtonian fluids regarding the thin film are mainly used in wire coating, paintings and fiber coatings, removal of foodstuff objects, numerous freezing subjects, container fluidization, and sheet coatings. To see the provisions used on behalf of all these applications, many researchers focused on studying the flow of thin liquid film over rigid and stretched surfaces.

Andersson et al. [1] have examined the Power-law fluid thin film flow affected through the time-dependent extending medium using similarity alteration. Myers [2] studied Power-law fluid flow considering small shear forces. The key features of his study are to investigate the influence of the modeled parameters over the thin film of the Power-law liquid when the shear rate approaches zero. Ali et al. [3] inspected the flow of the liquid film, considering the micropolar fluid flow using the extending surface. Similarly, Abbas et al. [4] examined the liquid film motion of the viscoelastic liquid using the extending surface. Siddiqui et al. [5] considered the liquid film flow 
using the two non-Newtonian fluids over a vertical surface. They used the analytical technique to obtain the results of the model problem. Li et al. [6] examined the nanofluid streaming on a thin layer liquid including thermal radiations. Dandapat et al. [7] examined the film flow considering the Marangoni convection and the biviscosity terminology. In addition to nonlinear free surface boundary conditions, complete expression of Navier-Stokes and continuity are analytically solved. Gul et al. [8] discussed the model of effective Pr number for thin film flow of nanofluids over stretching sheet.

Rudraiah et al. [9] are the pioneer to introduce the Marangoni convection concept in the fluid flow considering the open cavity. Chen et al. [10] have explored Marangoni convection's effect considering the Power-law model liquid film flow past an unstable surface. They observed the influence of the various parameters in the existence of the surface tension. Wang [11], Narayana and Sibanda [12], and Qasim et al [13] have examined the liquid film flow over a stretched surface. They have used the unsteady flow using the same similarity transformations. Gul et al. [14] have extended the idea of the liquid film flow by adding the Marangoni convection influence over the liquid film flow. Vajravelu et al. [15] explored the mathematical model for the thin layer streaming of the Power-law liquid over an unstable extending and permeable surface. Noor et al. [16] have analyzed thin-film liquid properties in thermos capillary and magnetic fields. Similarly, Shah et al. [17] investigated the fluid's convective flow over an extending surface.

It is important to remember that, in the case of nonNewtonian fluids, the interaction between the deformation rate and shear forces is not linear. The Carreau fluid's extreme shear ratio functions as the Power-law fluid, and the Carreau fluid behaves as Newtonian as the modest shear ratio. To find the mathematical results of the problem, they used the bvp4c scheme. The thin-film flow of the Carreau fluid over an inclined surface was scrutinized by Tshehla [18]. Akbar et al. [19] explored the model of Carreau fluid flow across a porous surface. Using a curved channel, Abbasi et al. [20] examined the peristaltic transport of the Carreau fluid. Khellaf and Lauriat [21] have examined the same fluid flow among the double concentric cylinders. Myers et al. [2] studied the liquid film flow considering Power-law, Ellis, and Carreau models. The related and interesting work can also be seen in the existing literature $[22,23]$.

The general Newtonian Carreau liquid remained was first offered by Carreau [24]. The model turns sensibly fit through the interruptions of polymers' performance in numerous stream positions. Mostly, the Carreau model is well-matched for certain diluted polymer results and meltdown. It determines the behavior of many nonNewtonian liquids to incorporate shear thinning and shear thickening. The Carreau fluid model was subsequently determined by numerous investigators, as its uses in many natural and then scientific innovations. Akbar et al. [19] found the double resolutions of magneto-hydrodynamics stagnation point stream of Carreau liquid near a porous stretching surface. Abbasi et al. [20], Khan et al. [25], and Iqbal et al. [26] discussed the thermodynamics of a Carreau fluid tinny film using different geometries. Kohilavani et al. [27] measured the flow of thin layer plus energy transmission in the Carreau liquid over a porous extending surface. Similarly, Sarada et al. [28], Gowda et al. [29], and Makinde et al. [30] examined the simultaneous convective flow of Carreau fluid, the behavior of non-Newtonian fluids, and ferromagnetic nanofluid flow over stretching surfaces along with MHD and thermal radiation effects.

In view of the above interesting debate, the contribution of this analysis is to extend the existing study of thin layer flow of the Carreau fluid [25] including the following steps.

(i) The couple stress fluid is included to extend the published work [25]

(ii) The previous work [25] is extended with the addition of the magnetic field

(iii) also includes the influence of Marangoni convection

\section{Problem Formulation}

The mathematical model has been focused on the two-dimensional thin film flow considering Carreau fluid. The liquid film's width $h(t)$ is uniform, and the surface stretches across the $x$-axis, as illustrated in Figure 1 . The $y$-axis is considered vertical to the $x$-axis and in the direction of the liquid film's thickness. The thin film is stretched with the $U_{w}(x, t)=b x(1-\alpha t)^{-1}$ (uniform velocity), where $\alpha$ and $b$ are constants and $b>0$ shows the stretching rate. Here, $t$ is the time and $\alpha$ is the constant used in the unsteadiness parameter. $V_{w}=\sqrt{(v b /(1-\alpha t))}$ is the suction/injection velocity such that $V_{w}=0, V_{w}>0$, and $V_{w}<0$ belong to impermeability, suction, and injection cases, respectively. The surface temperature is symbolized by $T_{w}$, and thin film thickness is $h(t)$. Since, in the above mathematical modeling, we considered the Carreau fluid flowing past an extending hot plate, the expression of $\tau$ (Cauchy stress tensor) for the said fluid is known as [27]

$$
\tau=-I p+\varepsilon\left(A_{1}\right) .
$$

Here,

$$
\varepsilon=\varepsilon_{\infty}+\left[1+(\lambda \dot{\gamma})^{2}\right]^{((n-1) / 2)}\left(\varepsilon_{0}-\varepsilon_{\infty}\right) .
$$

Here, $\varepsilon$ demonstrates the apparent viscosity, $\varepsilon_{\infty}, p, \tau$, and $\varepsilon_{0}$ denote the infinite-shear-rate viscosity, the pressure term, the Cauchy stress tensor, and zero-shear rate viscosity, respectively, $I$ used to show identity tensor, $\lambda$ is the material time constant, and $n$ is the power-law index. The shear rate is defined as

$$
\dot{\gamma}=\sqrt{\frac{1}{2} \sum_{i} \sum_{j} \dot{\gamma}_{i j} \dot{\gamma}_{j i}}
$$

and $\tau$ is defined as [27] 


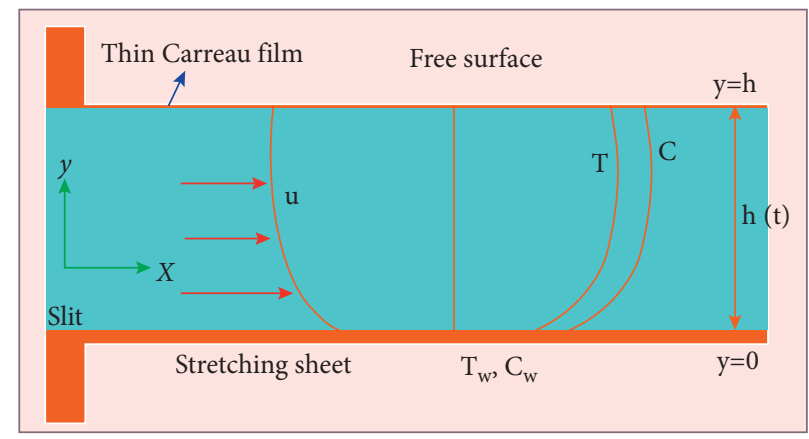

Figure 1: Coordinate system and physical flow model.

$$
\tau=-p I+A_{1} \varepsilon_{0}\left[(\lambda \dot{\gamma})^{2}+1\right]^{((n-1) / 2)} .
$$

The power-law index falls between $0<n<1$, and the Carreau model shows the shear-thinning or pseudoplastic properties. The dilatants' behavior accurses when $n>1$. The magnetic field is considered in the transverse direction in the unsteady form such as $B(t)=B_{0}(1-a t)^{-0.5}$. About the Carreau fluid model, the liquid film flow can be compiled as

$$
\frac{\partial u}{\partial x}+\frac{\partial v}{\partial y}=0
$$

Momentum equation is

$$
\begin{aligned}
\frac{\partial u}{\partial t}+u \frac{\partial u}{\partial x}+v \frac{\partial v}{\partial y}= & v\left[1+\lambda^{2}\left(\frac{\partial u}{\partial y}\right)^{2}\right]^{((n-1) / 2)}\left(\frac{\partial^{2} u}{\partial y^{2}}\right)+v \lambda^{2}(n-1)\left(\frac{\partial^{2} u}{\partial y^{2}}\right)\left[1+\lambda^{2}\left(\frac{\partial u}{\partial y}\right)^{2}\right]^{((n-3) / 2)}\left(\frac{\partial u}{\partial y}\right)^{2} \\
& -\frac{\sigma B^{2}(t) u}{\rho_{f}}-\frac{\eta^{*}}{\rho_{f}} \frac{\partial^{4} u}{\partial y^{4}}
\end{aligned}
$$

Heat/energy equation is

$$
\begin{aligned}
\frac{\partial T}{\partial t}+u\left(\frac{\partial T}{\partial x}\right)+v\left(\frac{\partial T}{\partial y}\right)-\frac{\kappa}{\left(\rho c_{p}\right)}\left(\frac{\partial^{2} T}{\partial y^{2}}\right) & =\tau\left[D_{B} \frac{\partial C}{\partial y} \frac{\partial T}{\partial y}+\frac{D_{T}}{T_{\infty}}\left(\frac{\partial T}{\partial y}\right)^{2}\right] \\
\frac{\partial C}{\partial t}+u\left(\frac{\partial C}{\partial x}\right)+v\left(\frac{\partial C}{\partial y}\right)-D_{B}\left(\frac{\partial^{2} C}{\partial y^{2}}\right) & =\left(\frac{D_{T}}{T_{\infty}}\right)\left(\frac{\partial^{2} T}{\partial y^{2}}\right)
\end{aligned}
$$

where the element of velocity $(u, v)$ varies along the $x$ and $y$ direction, $\lambda$ represents the material time constant, $\rho$ denotes density, $\kappa$ shows thermal conductivity, $c_{p}$ is the capacity specific heat, and $T$ is temperature of fluid.
The surface tension varies in a linear way, depending on the temperature. The other is made unchanged by the other properties of the fluid. Surface tension is described as follows [7, 11-14]: 
According to the abovementioned assumptions, the physical conditions are

$$
\begin{aligned}
& \text { at } y=0, \\
& u=U_{w}, \\
& v=V_{w}, \\
& C=C_{w}, \\
& T=T_{w}, \\
& \text { at } y=h(t), \\
& \left(\frac{\partial u}{\partial y}\right)=0, \\
& \frac{\partial C}{\partial y}=0=\frac{\partial T}{\partial y}, \\
& v=\frac{\partial h}{\partial t}
\end{aligned}
$$

$$
C_{w}=C_{0}-C_{\text {ref }}\left(\frac{b x^{2}}{2 v}\right)(1-\alpha t)^{-(3 / 2)}
$$

where $T_{0}$ and $C_{0}$ and $T_{\text {ref }}$ and $C_{\text {ref }}$ indicate the slit and reference temperature and concentration, respectively.
$T_{\text {ref }}$ and $C_{\text {ref }}$ can be treated as a constant or differences of a uniform temperature and concentration. The concept of $T_{w}$ impersonates the situation in which the temperature of the sheet reduces in proportion to $x^{2}$ from $T_{0}$ at the slit; also, the quantity of thermal drop along the surface of the sheet improves over time.

Next, we introduce the similarity factors as in [16]:

$$
\begin{aligned}
& \psi=\sqrt{\frac{v b}{1-\alpha t}} x f(\eta) \\
& u=\frac{\partial \psi}{\partial y}=\frac{b x}{1-\alpha t} f^{\prime}(\eta) \\
& v=-\frac{\partial \psi}{\partial x}=-\sqrt{\frac{v b}{1-\alpha t}} f(\eta) \\
& \eta=y \sqrt{\frac{b}{v(1-\alpha t)}} \\
& \beta=h \sqrt{\frac{b}{v(1-\alpha t)}}, \\
& \frac{\mathrm{d} h}{\mathrm{~d} t}=\frac{-\alpha \beta}{2} \sqrt{\frac{v}{b(1-\alpha t)}}, \\
& T=T_{0}-T_{\text {ref }}(1-\alpha t)^{-(3 / 2)}\left(\frac{b x^{2}}{2 v}\right) \theta(\eta), \\
& C=C_{0}-C_{\mathrm{ref}}(1-\alpha t)^{-(3 / 2)}\left(\frac{b x^{2}}{2 v}\right) \phi(\eta) .
\end{aligned}
$$

By using equations (13) and (5), it is satisfied identically but equations (6)-(8) and (11) take the following form:

$$
\begin{aligned}
{\left[1+w_{e}^{2} f^{\prime \prime 2}\right]^{((n-3) / 2)}\left[1+\left(w_{e}^{2}\right) f^{\prime \prime 2}\right] f^{\prime \prime \prime}+\left[f f^{\prime \prime}-f^{\prime 2}-M f^{\prime}-\frac{S}{2}\left(f^{\prime}+\frac{\eta}{2} f^{\prime \prime}\right)\right]-K f^{v} } & =0, \\
\theta^{\prime \prime}+\operatorname{Pr}\left[f \theta^{\prime}-2 f^{\prime} \theta+N_{T} \theta^{\prime 2}+N_{B} \phi^{\prime} \theta^{\prime}-\frac{S}{2}\left(3 \theta-\eta \theta^{\prime}\right)\right] & =0, \\
\phi^{\prime \prime}+\left[f \phi^{\prime}-\frac{S}{2}\left(3 \phi-\eta \phi^{\prime}\right)-2 f^{\prime} \phi\right] S c+\left(\frac{N_{T}}{N_{B}}\right) \theta^{\prime \prime} & =0, \\
f(0) & =\delta, \\
f^{\prime}(0) & =1, \\
\theta(0) & =1=\phi(0), \\
f(\beta) & =\frac{S \beta}{2}, \\
f^{\prime \prime}(\beta) & =0, \\
\theta^{\prime}(\beta) & =\phi^{\prime}(\beta)=0,
\end{aligned}
$$


where

$$
\begin{aligned}
& M=\frac{\sigma B_{0}^{2}}{\rho b}, \\
& K=\frac{\eta^{*} b^{3}}{U^{w} \rho_{f}}, \\
& S=\frac{\alpha}{b}, \\
& \tau=\frac{(\rho c p)_{p}}{(\rho c p)_{f}}, \\
& \operatorname{Pr}=\frac{\mu c p}{k}, \\
& N_{B}=\frac{\tau\left(C_{w}-C_{\infty}\right) D_{B}}{v}, \\
& N_{T}=\frac{\tau\left(T_{w}-T_{\infty}\right) D_{T}}{v T_{\infty}} \\
& \mathrm{Sc}=\frac{v}{D_{B}}, \\
& W_{e}^{2}=\frac{\lambda^{2} b U_{w}^{2}}{v}, \\
& \delta=\frac{V_{w}}{\sqrt{v b}}
\end{aligned}
$$

where $M, S, \operatorname{Pr}, N_{T}, w_{e}^{2}, N_{B}, \mathrm{Sc}, \tau, K$, and $\operatorname{Re}_{x}$ are the magnetic field parameter, unsteady factor, Prandtl number, thermophoresis factor, local Weissenberg number, Brownian motion factor, Schmidt number, Cauchy stress tensor, couple stress parameter of the fluid, and local Reynolds number. $\delta$ is the suction $\backslash$ injection parameter.

In this model problem, the physical quantities of practical importance are $C_{f x}, \mathrm{Nu}_{x}$, and $\mathrm{Sh}_{x}$ as follows [7, 15]:

$$
\begin{aligned}
C_{f x} & =\frac{\tau_{w}}{\rho u_{w}^{2}} \\
\mathrm{Nu}_{x} & =\frac{q_{w} x}{k\left(T_{w}-T_{0}\right)} \\
\mathrm{Sh}_{x} & =\frac{q_{m} x}{k\left(C_{w}-C_{0}\right)},
\end{aligned}
$$

where $\tau_{w}, q_{w}$, and $q_{m}$ are represented by the below equations:

$$
\begin{aligned}
\tau_{w} & =\left.\mu_{0} \frac{\partial u}{\partial y}\left[1+\lambda^{2}\left(\frac{\partial u}{\partial y}\right)^{2}\right]^{((n-1) / 2)}\right|_{y=0}, \\
q_{w} & =-\left.k\left(\frac{\partial T}{\partial y}\right)\right|_{y=0}, \\
q_{m} & =-\left.k\left(\frac{\partial C}{\partial y}\right)\right|_{y=0}, \\
\operatorname{Re}_{x}^{(1 / 2)} C_{f x} & =\left\{w e^{2}\left(f^{\prime \prime}(0)\right)^{2}+1\right\}^{((n-1) / 2)} f^{\prime \prime}(0), \\
\operatorname{Re}_{x}^{-(1 / 2)} \mathrm{Nu}_{x} & =-\theta^{\prime}(0), \\
\operatorname{Re}_{x}^{-(1 / 2)} \operatorname{Sh}_{x} & =-\phi(0) .
\end{aligned}
$$

\section{HAM Solution}

The analytical approach has been used to examine the modeled problem. This technique includes the latest version BVPh 2.0 package of the Homotopy Analysis Method (HAM). BVPh 2.0 package tends to converge rapidly, and more iterations are possible in this method in a short time. The recent solution has been achieved using the package mentioned above up to the 30 order estimations. The trail solution for the modeled problem is obtained as

$$
\begin{aligned}
f_{0}(\eta) & =\frac{3}{2 \beta^{3}}[2 \beta+2 \delta-S \beta]\left(\frac{\eta^{3}}{6}-\frac{\eta^{2} \beta}{2}\right) \\
& +\eta+\delta, \\
\theta_{0}(\eta) & =1, \\
\phi_{0}(\eta) & =1, \\
L_{f}(f) & =\frac{\partial^{4}}{\partial \eta^{4}}, \\
L_{\theta}(\theta) & =\frac{\partial^{2}}{\partial \eta^{2}}, \\
L_{\phi}(\phi) & =\frac{\partial^{2}}{\partial \eta^{2}}, \\
L_{f}\left[A_{1}+A_{2} \eta+A_{3} \eta^{2}+A_{4} \eta^{4}\right] & =0 \\
L_{\theta}\left[A_{5}+A_{6} \eta\right] & =0, \\
L_{\phi}\left[A_{7}+A_{8} \eta\right] & =0,
\end{aligned}
$$


where $A_{n}(n=1,2, \ldots, 8)$ are the constants of the integration. The residual errors considering average estimations up to the $k^{\text {th }}$ order is defined as

$$
\begin{aligned}
\varepsilon_{k}^{f}\left(h_{f}\right) & =\frac{1}{L+1} \sum_{J=0}^{L}\left[\sum_{m=0}^{k}\left(f_{m}\right)_{\eta}=j \pi \eta\right]^{2}, \\
\varepsilon_{k}^{\theta}\left(h_{f}, h_{\theta}\right) & =\frac{1}{L+1} \sum_{J=0}^{L}\left[\sum_{m=0}^{k}\left(f_{m}\right)_{\eta}=j \pi \eta, \sum_{m=0}^{k}\left(\theta_{m}\right)_{\eta}=j \pi \eta\right]^{2}, \\
\varepsilon_{k}^{\theta}\left(h_{f}, h_{\theta}, h_{\phi}\right) & =\frac{1}{L+1} \sum_{J=0}^{L}\left[\sum_{m=0}^{k}\left(f_{m}\right)_{\eta}=j \pi \eta, \sum_{m=0}^{k}\left(\theta_{m}\right)_{\eta}=j \pi \eta, \sum_{m=0}^{k}\left(\phi_{m}\right)_{\eta}=j \pi \eta\right]^{2}, \\
\varepsilon^{t} & =\varepsilon_{k}^{f}+\varepsilon_{k}^{\theta}+\varepsilon_{k}^{\phi} .
\end{aligned}
$$

The sum of the total residual errors $\varepsilon_{m}^{t}$ has been attained from the fluid velocity, temperature, and concentration profiles.

\section{Result and Discussion}

The objectives of the current portion are to inspect the effect of major factors on the thin liquid flow of Carreau liquid through an extending sheet. The findings obtained for the velocity, heat, and concentration profiles $\left(f^{\prime}(\eta), \theta(\eta)\right.$, and $\phi(\eta))$ are seen in plots to discuss the effects of prevailing factors such as $K, M, S$, we ${ }^{2}, N_{T}, N_{B}, \operatorname{Pr}$, and Sc. For computational estimates, we assumed $(K=0.6, M=0.6$, $S=0.4, \quad$ we $^{2}=1, \quad N_{T}=0.8, \quad N_{B}=0.7, n=0.1$, $\operatorname{Pr}=10.4, \Psi=1$, and $\mathrm{Sc}=0.4$ ). But these values are considered to be consistent in the analysis, except that the variable values are seen in the corresponding figures and tables. The coordinate system and physical geometry of the model problem are represented in Figure 1. The influence of $M$ on $f^{\prime}(\eta)$ (fluid velocity field) is presented in Figure 2. The velocity profile $f^{\prime}(\eta)$ has shown the decline behavior by increasing the magnitude of the magnetic parameter $M$. With increasing strength of the magnetic field, we will encounter a resistive force known as Lorentz force. The rate of Lorentz force increases with a greater value of magnetic parameter. This force behaves as an agent of resistance to fluid motion. Due to this fact, the speed of the liquid film declined. The effect of $S$ (unsteady factor) on $f^{\prime}(\eta)$ (velocity field) is presented in Figure 3. We witnessed from the plot a decrease in velocity fields for rising magnitude of $S$. Obviously, for the greater degree of the $S$ unsteadiness factor, the density of the thin film rises, and hence, the fluid velocity declines with maximum value of $S$. Figure 4 indicates another major effect of we $e^{2}$ on thin layer liquid $f^{\prime}(\eta)$ velocity. We observed a drop in $f^{\prime}(\eta)$ from the plot for the increasing amount of $\mathrm{we}^{2}$. In this graph, we can see contradictory patterns occurring with various values of $\mathrm{we}^{2}$ on fluid velocity. In case of shear-thinning, the velocity of the liquid thin film reduces, whenever we strengthen its value $\mathrm{we}^{2}$. In comparison, the behavior of $\mathrm{we}^{2}$ is opposite for shear thickening fluid. This has been confirmed that the boundary film width reduces for $0<n<1$, although it improves for $n>1$. Figure 5 illustrates the importance of $K$ (couple stress factor) on the $f^{\prime}(\eta)$ (velocity profile). Here, as the magnitude of the couple stress factor increases, the $f^{\prime}(\eta)$ velocity profile is reduced. It is interesting that the liquid becomes a Newtonian fluid when the couple stress factor tends to zero $(K \longrightarrow 0)$. Figure 6 highlights influence of $\beta$ (layer thickness factor) on the $f^{\prime}(\eta)$ velocity profiles of the fluid film. A rise in the value of $f^{\prime}(\eta)$ depreciation in the fluid flow velocity profiles has been observed. Physically, the viscosity of the momentum boundary film is enlarged by the growth of the value of $\beta$. For this purpose, we observed a depreciation in fluid velocity. The influence of $N_{T}$ (thermophoresis factor) is captured in Figures 7 and 8 on $\theta(\eta)$ (temperature field) and $\phi(\eta)$ (concentration field). Therefore, the conclusion is taken from Figure 7 that $\theta(\eta)$ is growing with rising values $N_{T}$. Figure 8 illustrates the influence of $N_{T}$ on $\phi(\eta)$, where an increasing behavior is observed in $\phi(\eta)$ with the growing quantity of $N_{T}$. The impact of $N_{B}$ on $\theta(\eta)$ (thermal profile) and $\phi(\eta)$ (concentration field) is shown in Figures 9 and 10. Figure 9 indicates that $\theta(\eta)$ grows with the rising values $N_{B}$. Brownian motion particles spontaneously travel across the fluid, and therefore, increase in $N_{B}$ produces an improvement in the fluid objects' random motion and as a result more heat can be transported. But at the other side, in the case of $\phi(\eta)$ Figure 10, the opposite behavior is noticed. Figure 11 demonstrates the influence of $\operatorname{Pr}$ on the $\theta(\eta)$ (temperature filed) of thin film fluid. It is shown from this graph that, with the rising values of $\operatorname{Pr}, \theta(\eta)$ indicates a decrease. Physically, with the increasing values of $\mathrm{Pr}$, decline the temperature field for its larger values. Figure 12 examines the influence of Sc on the $\phi(\eta)$ field. It is clearly found from the graph that $\phi(\eta)$ and its corresponding width of the boundary layer are decreasing function of Sc. Also, Sc is inversely related to the coefficient of diffusion. Therefore, a rise in Sc leads to a lower coefficient of diffusion. So, a 


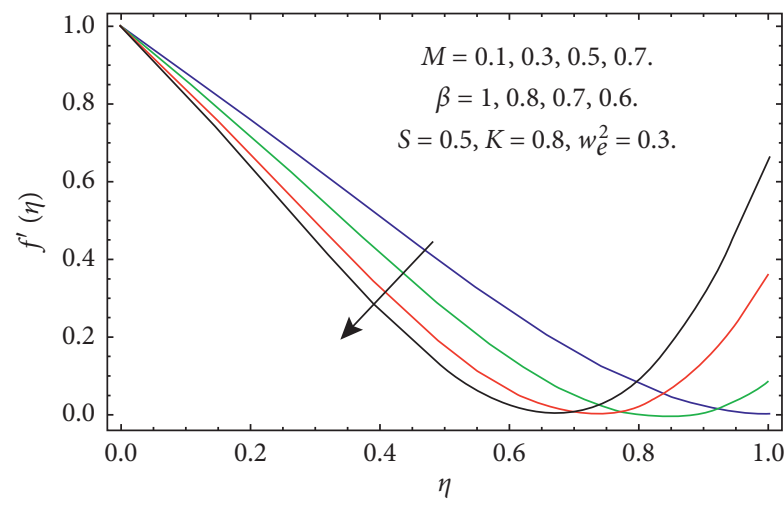

Figure 2: $f^{\prime}(\eta)$ (velocity field) for multiple values of $M$.

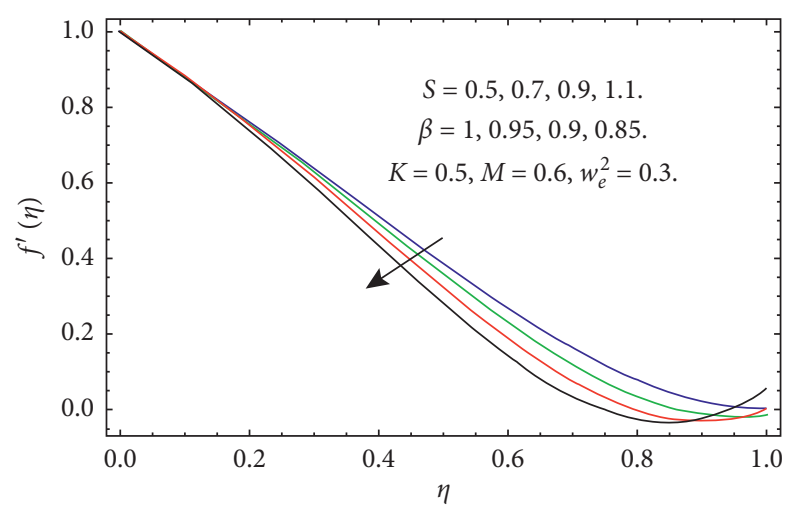

Figure 3: $f^{\prime}(\eta)$ (velocity field) for multiple values of $S$.

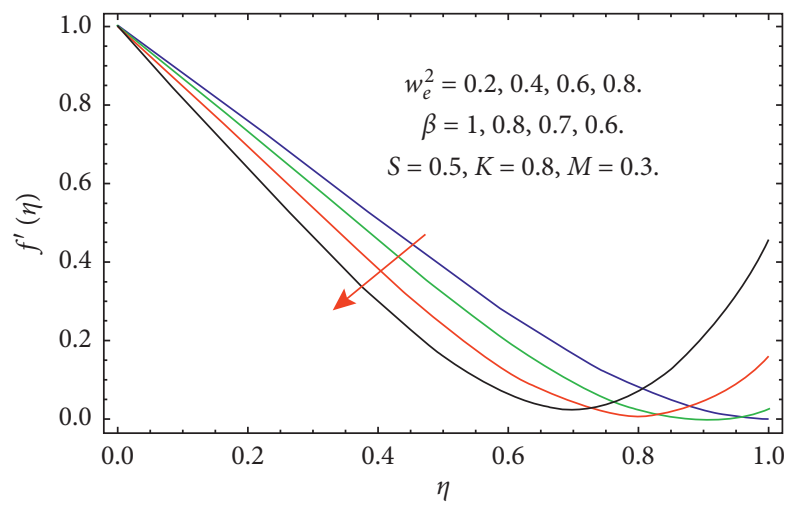

FIGURE 4: $f^{\prime}(\eta)$ (velocity field) for multiple values of $\mathrm{We}^{2}$.

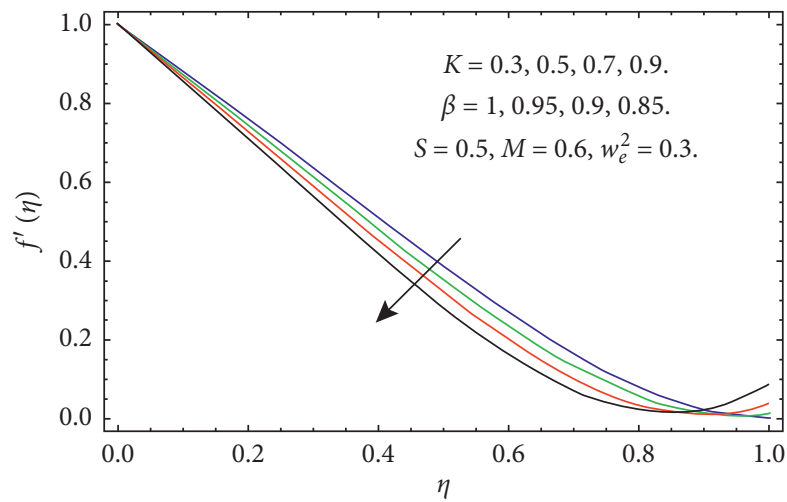

Figure 5: $f^{\prime}(\eta)$ (velocity field) for multiple values of $K$.

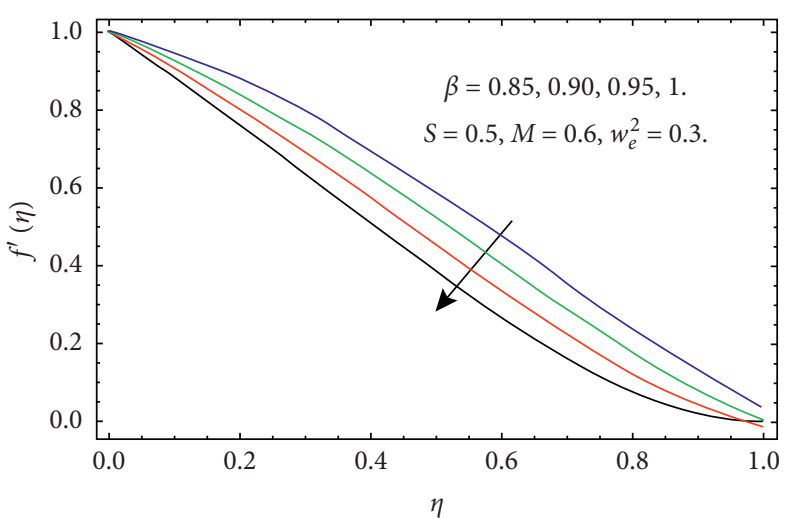

Figure 6: $f^{\prime}(\eta)$ (velocity field) for multiple values of $\beta$.

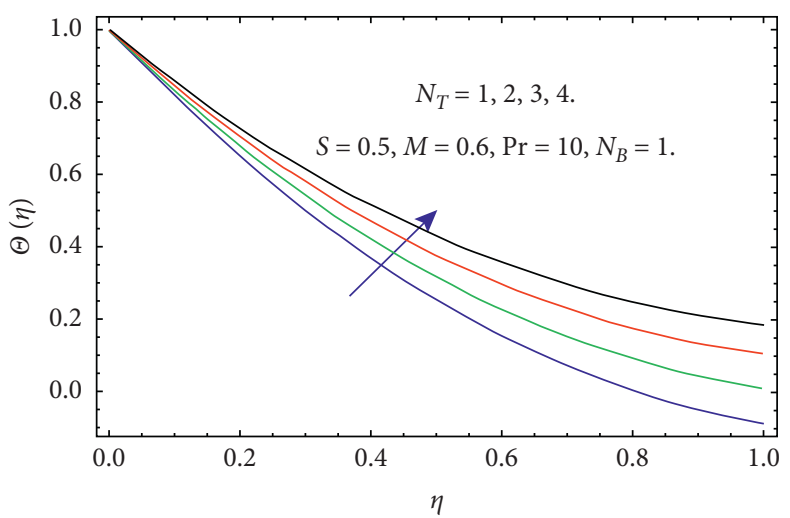

Figure 7: $\Theta(\eta)$ (temperature field) for multiple values of $N_{T}$.

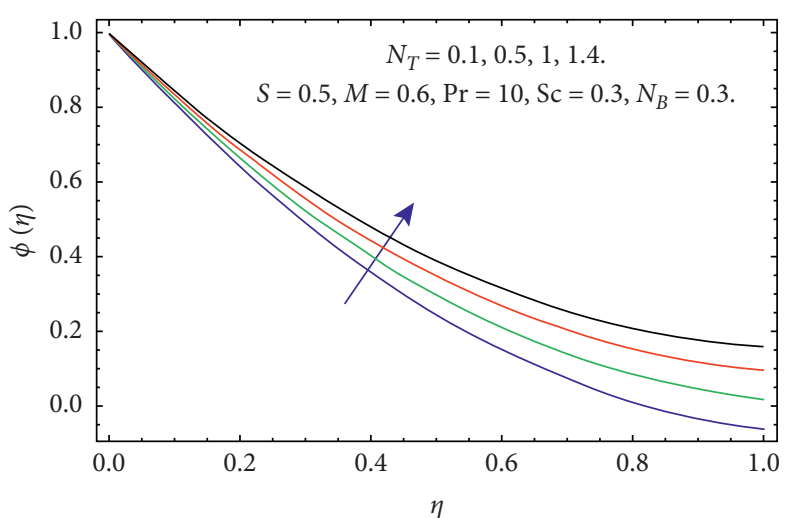

Figure 8: $\phi(\eta)$ (concentration field) for multiple values of $N_{T}$.

decrease in the $\phi(\eta)$ profile is caused by such a lower diffusion coefficient. The skin friction of the Carreau liquid film increasing with the larger magnitude of the parameters $M, n, S, k$, and we is shown in Table 1 . The resistance force enlarges due the increasing magnitude of these parameters, and consequently, the skin friction boots up. Similarly, the thermophoretic parameter and Brownian motion parameter improve the heat transfer rate for the larger values of these parameters, as shown in Table 2, while the increasing value of the unsteady parameter reduces the heat transfer rate. 


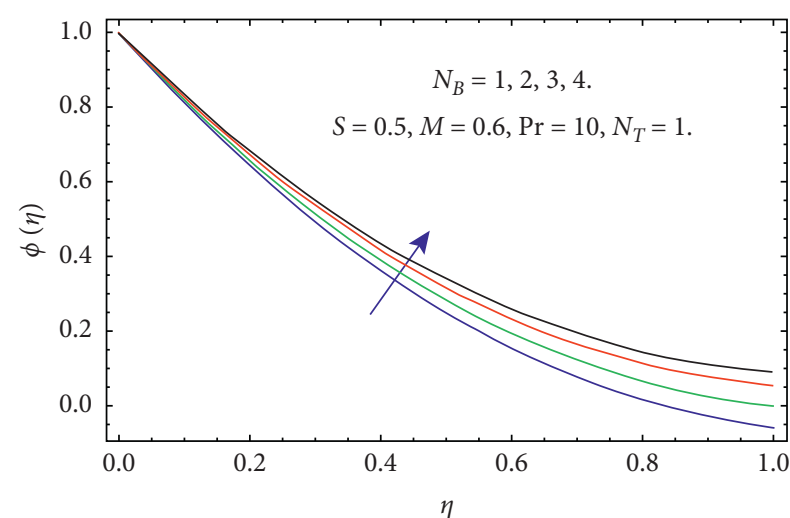

Figure 9: $\Theta(\eta)$ (temperature field) for multiple values of $N_{B}$.

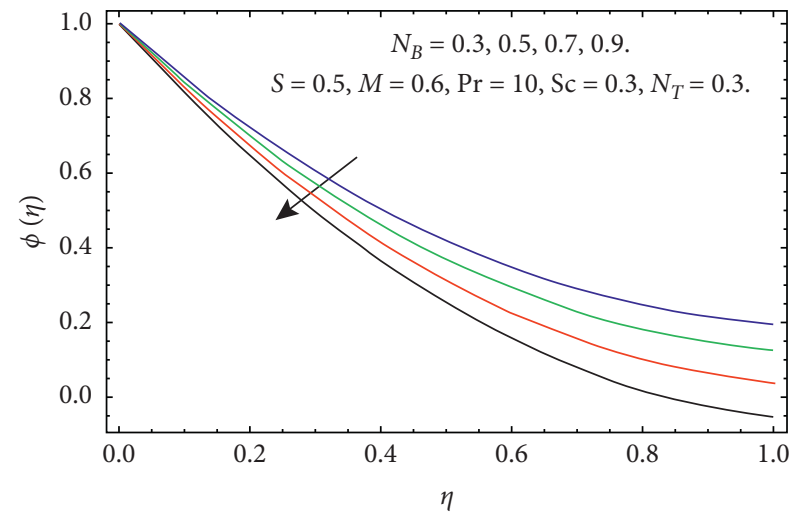

Figure 10: $\phi(\eta)$ (concentration field) for multiple values of $N_{B}$.

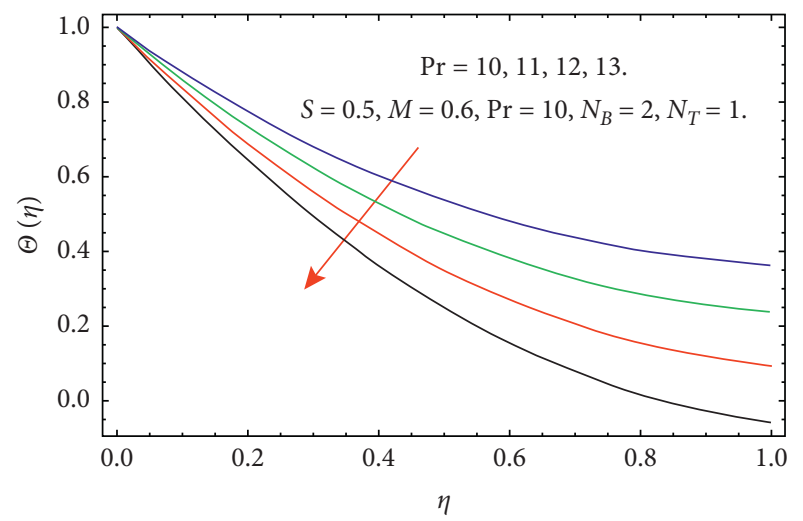

Figure 11: $\Theta(\eta)$ (temperature field) for multiple values of Pr.

$\mathrm{Nt}, \mathrm{Nb}$, and $S$ augmenting the Sherwood number for the increasing amount of these parameters is displayed in Table 3, but the parameter Sc reduces the mass transfer rate for its larger values. The comparison of the present study and published work [31] is shown in Table 4.

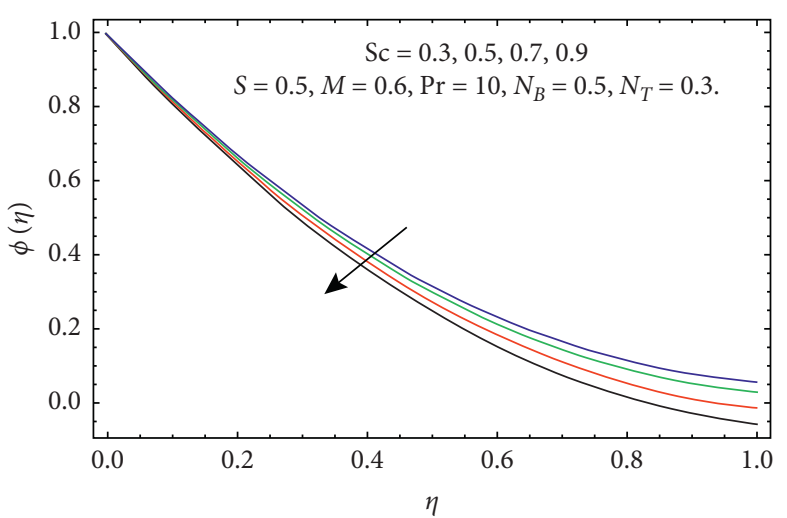

Figure 12: $\phi(\eta)$ (concentration field) for multiple values of Sc.

TABLe 1: Skin friction versus embedded parameters.

\begin{tabular}{ccccccc}
\hline$M$ & We & $k$ & $S$ & $n$ & $\Psi$ & $\operatorname{Re}_{x}^{(1 / 2)} C_{f x}$ \\
\hline 0.1 & 0.1 & 0.1 & 0.1 & 0.1 & 0.1 & 3.41518 \\
0.2 & & & & & & 3.4223 \\
0.3 & & & & & & 3.42942 \\
& 0.2 & & & & & 3.5927 \\
& 0.3 & & & & & 3.89123 \\
& & 0.2 & & & & 4.97121 \\
& 0.3 & & & & 4.36927 \\
& & 0.2 & & & 4.70292 \\
& & 0.3 & & & 4.78074 \\
& & & 0.2 & & 4.85167 \\
& & & 0.3 & & 0.2 & 4.99909 \\
& & & & & 0.3 & 5.15497 \\
\hline
\end{tabular}

TABLE 2: Nusselt number versus embedded parameters.

\begin{tabular}{cccc}
\hline$N_{T}$ & $N_{B}$ & $S$ & $\mathrm{Re}_{x}^{(-1 / 2)} \mathrm{Nu}_{x}$ \\
\hline 0.1 & 0.1 & 0.1 & 1.02978 \\
0.2 & & & 1.241424 \\
0.3 & & & 1.452541 \\
& 0.2 & & 1.53642 \\
& 0.3 & 0.2 & 1.64861 \\
& & 0.3 & 1.352101 \\
& & & 1.053761 \\
\hline
\end{tabular}

TABle 3: Sherwood number versus embedded parameters.

\begin{tabular}{ccccc}
\hline$N_{T}$ & $N_{B}$ & $S$ & $\mathrm{Sc}$ & $\mathrm{Re}_{x}^{(-1 / 2)} \mathrm{Sh}_{x}$ \\
\hline 0.1 & 0.1 & 0.1 & 0.1 & 0.9243210 \\
0.2 & & & & 0.8233101 \\
0.3 & & & & 0.7123013 \\
& 0.2 & & & 1.0234124 \\
& 0.3 & 0.2 & & 1.1342151 \\
& & 0.3 & & 1.3284632 \\
& & & 0.2 & 1.4122154 \\
& & & 0.3 & 1.13243702 \\
& & & &
\end{tabular}


TABLE 4: Comparison with published work [27] considering common parameters.

\begin{tabular}{cccccc}
\hline$N_{T}$ & $N_{B}$ & $\operatorname{Re}_{x}^{(-1 / 2)} \mathrm{Nu}_{x}$ present & $\mathrm{Re}_{x}^{(-1 / 2)} \mathrm{Nu}_{x}[19]$ & $\mathrm{Re}_{x}^{(-1 / 2)} \mathrm{Sh}_{x} \operatorname{present}$ & $\mathrm{Re}_{x}^{(-1 / 2)} \mathrm{Sh}_{x}[19]$ \\
\hline 0.1 & 0.1 & 1.02978 & 1.029421 & 0.9243210 & 0.9244322 \\
0.2 & & 1.241424 & 1.2413621 & 0.8233101 & 0.82342122 \\
0.3 & 1.452541 & 1.4526312 & 0.7123013 & 0.7123124 \\
& 0.2 & 1.53642 & 1.536321 & 1.0234124 & 1.0234232 \\
& 0.3 & 1.64861 & 1.6483521 & 1.1342151 & 1.1343241 \\
\hline
\end{tabular}

\section{Conclusions}

In this analysis, by taking into account the influence of Marangoni convection, couple stress, and MHD, we investigated the flow, heat transport and concentration properties of thin film fluid flow of the Carreau fluid model over an unsteady stretching sheet. The Buongiorno model was also used to develop the effects of thermophoresis and Brownian diffusion. The key finding of the present analysis is taken in the following way.

(i) The obtained results shows that the velocity field is dependent on the thickness parameter $\beta$. The convergence phenomenon is validated with the variation of the thickness parameter.

(ii) The velocity field declines with the augmentation of the thickness parameter $\beta$.

(iii) The increasing value of the couple stress parameter $K$ improves the resistive force and consequently the fluid motion decline.

(iv) $f^{\prime}(\eta)$ decelerates with increasing values of $M, S$, and $\mathrm{we}^{2}$.

(v) The thermal field $\theta(\eta)$ reduces by rising $\operatorname{Pr}$ whereas it strengthens for $N_{B}$ and $N_{T}$.

(vi) Whereas the opposite pattern for both $N_{B}$ and $N_{T}$, it is observed on $\phi(\eta)$ profile.

(vii) Both the concentration and related layer thickness are the decreasing function of Sc.

Symbol description

\section{Nomenclature}

$u$ and $v: \quad x$ and $y$ components of velocity $\left(\mathrm{ms}^{-1}\right)$

$U_{w}(x, t)$ : Stretching surface velocity $\left(\mathrm{ms}^{-1}\right)$

$h(t): \quad$ Thickness of the fluid $(\mathrm{m})$

$\rho: \quad$ Density $\left(\mathrm{kgm}^{-3}\right)$

$c_{p}: \quad$ Specific heat capacity $\left(\mathrm{Jkg}^{-1} \mathrm{~K}^{-1}\right)$

$x$ and $y$ : Cartesian coordinates $(\mathrm{m})$

$\kappa: \quad$ Thermal conductivity

we $^{2}$ : Local Weissenberg number

$T_{w}: \quad$ Surface temperature $(\mathrm{K})$

$T_{0}: \quad$ Slit temperature $(\mathrm{K})$

$T_{\text {ref: }}: \quad$ Reference temperature $(\mathrm{K})$

$N_{B}$ : Brownian motion parameter

$n$ : $\quad$ Power-law index

$\eta_{0}: \quad$ Zero-shear rate viscosity $\left(\mathrm{kgm}^{-1} \mathrm{~s}^{-1}\right)$

p: $\quad$ Pressure $(\mathrm{Pa})$

$A_{1}: \quad$ Rivlin-Ericksen tensor $(\mathrm{Pa})$

\begin{tabular}{|c|c|}
\hline$\mu:$ & Dynamic viscosity $\left(\mathrm{kgm}^{-1} \mathrm{~s}^{-1}\right)$ \\
\hline$v:$ & Kinematic viscosity $\left(\mathrm{m}^{2} \mathrm{~s}^{-1}\right)$ \\
\hline$S:$ & Unsteady parameter \\
\hline Pr: & Prandtl number \\
\hline$\lambda:$ & Material time constant \\
\hline $\operatorname{Re}_{x}:$ & Reynolds number \\
\hline$T:$ & Temperature (K) \\
\hline$N_{T}:$ & Thermophoresis parameter \\
\hline$\tau:$ & Cauchy stress tensor (Pa) \\
\hline Sc: & Schmidt number \\
\hline M: & Magnetic parameter \\
\hline $\mathrm{Nu}_{x}:$ & Local Nusselt number \\
\hline$\varepsilon_{\infty}:$ & Infinite-shear-rate viscosity $\left(\mathrm{kgm}^{-1} \mathrm{~s}^{-1}\right)$ \\
\hline$I:$ & Identity tensor \\
\hline$\varepsilon:$ & Apparent viscosity $\left(\mathrm{kgm}^{-1} \mathrm{~s}^{-1}\right)$ \\
\hline$b:$ & Stretching rate $\left(\mathrm{s}^{-1}\right)$ \\
\hline$t:$ & Time (s) \\
\hline$\left(C_{f x}\right)$ & Skin-friction coefficient \\
\hline$\left(\mathrm{Sh}_{x}\right)$ & Sherwood number. \\
\hline
\end{tabular}

\section{Data Availability}

All the data exist in the manuscript.

\section{Conflicts of Interest}

The authors declare that they have no conflicts of interest.

\section{References}

[1] H. I. Andersson, J. B. Aarseth, N. Braud, and B. S. Dandapat, "Flow of a power-law fluid film on an unsteady stretching surface," Journal of Non-newtonian Fluid Mechanics, vol. 62, no. 1, pp. 1-8, 1996.

[2] T. G. Myers, "Application of non-Newtonian models to thin film flow," Physical review. E, Statistical, nonlinear, and soft matter physics, vol. 72, no. 6, Article ID 066302, 2005.

[3] V. Ali, T. Gul, S. Afridi, F. Ali, S. Alharbi, and I. Khan, "Thin film flow of micropolar fluid in a permeable medium," Coatings, vol. 9, no. 2, p. 98, 2019.

[4] Z. Abbas, T. Hayat, M. Sajid, and S. Asghar, "Unsteady flow of a second grade fluid film over an unsteady stretching sheet," Mathematical and Computer Modelling, vol. 48, no. 3-4, pp. 518-526, 2008.

[5] A. M. Siddiqui, M. Ahmed, and Q. K. Ghori, "Thin film flow of non-Newtonian fluids on a moving belt," Chaos, Solitons \& Fractals, vol. 33, no. 3, pp. 1006-1016, 2007.

[6] J. Li, L. Liu, L. Zheng, and B. Bin-Mohsin, "Unsteady MHD flow and radiation heat transfer of nanofluid in a finite thin film with heat generation and thermophoresis," Journal of the Taiwan Institute of Chemical Engineers, vol. 67, pp. 226-234, 2016. 
[7] B. S. Dandapat, B. Santra, and H. I. Andersson, "Thermocapillarity in a liquid film on an unsteady stretching surface," International Journal of Heat and Mass Transfer, vol. 46, no. 16, pp. 3009-3015, 2003.

[8] T. Gul, S. Nasir, S. Islam, Z. Shah, and M. A. Khan, "Effective Prandtl number model influences on the $\gamma \mathrm{Al}_{2} \mathrm{O}_{3}-\mathrm{H}_{2} \mathrm{O}$ and $\gamma \mathrm{Al} 2 \mathrm{O} 3-\mathrm{C} 2 \mathrm{H} 6 \mathrm{O} 2$ nanofluids spray along a stretching cylinder," Arabian Journal for Science and Engineering, vol. 44, pp. 1-16, 2018.

[9] N. Rudraiah, M. Venkatachalappa, and C. K. Subbaraya, "Combined surface tension and buoyancy-driven convection in a rectangular open cavity in the presence of a magnetic field," International Journal of Non-linear Mechanics, vol. 30, no. 5, pp. 759-770, 1995.

[10] C.-H. Chen, "Marangoni effects on forced convection of power-law liquids in a thin film over a stretching surface," Physics Letters A, vol. 370, no. 1, pp. 51-57, 2007.

[11] C. Y. Wang, "Liquid film on an unsteady stretching surface," Quarterly of Applied Mathematics, vol. 48, no. 4, pp. 601-610, 1990.

[12] M. Narayana and P. Sibanda, "Laminar flow of a nanoliquid film over an unsteady stretching sheet," International Journal of Heat and Mass Transfer, vol. 55, no. 25-26, pp. 7552-7560, 2012.

[13] M. Qasim, Z. H. Khan, R. J. Lopez, and W. A. Khan, "Heat and mass transfer in nanofluid thin film over an unsteady stretching sheet using Buongiorno's model," The European Physical Journal Plus, vol. 131, no. 1, pp. 1-11, 2016.

[14] T. Gul, M. Z. Ullah, A. K. Alzahrani, Z. Zaheer, and I. S. Amiri, "MHD thin film flow of kerosene oil based CNTs nanofluid under the Influence of Marangoni convection," Physica Scripta, vol. 95, no. 1, pp. 015702-015724, 2020.

[15] K. Vajravelu, K. V. Prasad, and C.-O. Ng, "Unsteady flow and heat transfer in a thin film of Ostwald-de Waele liquid over a stretching surface," Communications in Nonlinear Science and Numerical Simulation, vol. 17, no. 11, pp. 4163-4173, 2012.

[16] N. F. M. Noor and I. Hashim, "Thermocapillarity and magnetic field effects in a thin liquid film on an unsteady stretching surface," International Journal of Heat and Mass Transfer, vol. 53, no. 9-10, pp. 2044-2051, 2010.

[17] R. A. Shah, S. Rehman, M. Idrees, and T. Abbas, "Magnetohydrodynamic convection flow on an unsteady surface stretching with pressure-dependent transverse velocity and surface tension linearly varying with temperature," Heat Transfer Research, vol. 48, pp. 1-25, 2017.

[18] M. S. Tshehla, "The flow of a Carreau fluid down an incline with a free surface," International Journal of the Physical Sciences, vol. 6, no. 16, pp. 3896-3910, 2011.

[19] N. S. Akbar, S. Nadeem, R. U. Haq, and S. Ye, "MHD stagnation point flow of Carreau fluid toward a permeable shrinking sheet: dual solutions," Ain Shams Engineering Journal, vol. 5, no. 4, pp. 1233-1239, 2014.

[20] F. M. Abbasi, T. Hayat, and A. Alsaedi, "Numerical analysis for MHD peristaltic transport of Carreau-Yasuda fluid in a curved channel with Hall effects," Journal of Magnetism and Magnetic Materials, vol. 382, pp. 104-110, 2015.

[21] K. Khellaf and G. Lauriat, "Numerical study of heat transfer in a non-Newtonian Carreau-fluid between rotating concentric vertical cylinders," Journal of Non-newtonian Fluid Mechanics, vol. 89, no. 1-2, pp. 45-61, 2000.

[22] T. Hayat, A. Shafiq, M. Nawaz, and A. Alsaedi, "MHD axisymmetric flow of third grade fluid between porous disks with heat transfer," Applied Mathematics and Mechanics, vol. 33, no. 6, pp. 749-764, 2012.
[23] T. Hayat, A. Shafiq, and A. Alsaedi, "Hydromagnetic boundary layer flow of Williamson fluid in the presence of thermal radiation and Ohmic dissipation," Alexandria Engineering Journal, vol. 55, no. 3, pp. 2229-2240, 2016.

[24] P. J. Carreau, "Rheological equations from molecular network theories," Transactions of the Society of Rheology, vol. 16, no. 1, pp. 99-127, 1972.

[25] N. S. Khan, T. Gul, P. Kumam et al., "Influence of inclined magnetic field on Carreau nanoliquid thin film flow and heat transfer with graphene nanoparticles," Energies, vol. 12, no. 8, p. 1459, 2019.

[26] K. Iqbal, J. Ahmed, M. Khan, L. Ahmad, and MehtibAlghamdi, "Non uniqueness solutions for the thin Carreau film flow and heat transfer over an unsteady stretching sheet," Applied Physics A, vol. 126, pp. 105 2-13, 2020.

[27] K. Naganthran, I. Hashim, and R. Nazar, "Non-uniqueness solutions for the thin Carreau film flow and heat transfer over an unsteady stretching sheet," International Communications in Heat and Mass Transfer, vol. 117, Article ID 104776, 2020.

[28] K. Sarada, R. J. P. Gowda, I. E. Sarris, R. N. Kumar, and B. C. Prasannakumara, "Effect of magnetohydrodynamics on heat transfer behaviour of a non-Newtonian fluid flow over a stretching sheet under local thermal non-equilibrium condition," Fluid, vol. 6, no. 8, p. 264, 2021.

[29] R. J. Punith Gowda, R. Naveen Kumar, B. C. Prasannakumara, B. Nagaraja, and B. J. Gireesha, "Exploring magnetic dipole contribution on ferromagnetic nanofluid flow over a stretching sheet: an application of Stefan blowing," Journal of Molecular Liquids, vol. 335, Article ID 116215, 2021.

[30] O. D. Makinde, B. C. P. Kumara, G. K. Ramesh, and B. J. Gireesha, "Simultaneous convection of Carreau fluid with radiation past a convectively heated moving plate," Defect and Diffusion Forum, vol. 389, pp. 60-70, 2018.

[31] K. Naganthran, I. Hashim, and R. Nazar, "Non-uniqueness solutions for the thin Carreau film flow and heat transfer over an unsteady stretching sheet," International Communications in Heat and Mass Transfer, vol. 117, p. 104776, 2020. 